\title{
METODOLOGIAS E MEDIDAS PARA A MINIMIZAÇÃO DE RESÍDUOS EM UMA INDÚSTRIA MOVELEIRA
}

\author{
Thiara Reis Lopes ${ }^{1 *}$, Sheila Aparecida Fritsch ${ }^{1}$, Juliana Bortoli Rodrigues Mees ${ }^{1}$ \\ Programa de Pós-Graduação em Tecnologias Ambientais, Universidade Tecnológica Federal do Paraná, CEP 85884-000, \\ Medianeira - PR, Brasil.
}

*E-mail: thiaralop@gmail.com

\section{RESUMO}

Este estudo foi desenvolvido em uma indústria moveleira localizada no oeste do estado do Paraná. Apresenta-se a aplicação de metodologias para priorização e minimização de resíduos propostos pela EPA, sob o enfoque do controle na fonte e reciclagem interna. Foram identificados os resíduos prioritários de acordo com o Número de Prioridade (N.P.), e selecionados os dez primeiros itens para a proposição das medidas. Entre eles, o efluente resina uréia-formol, o pó e a serragem de MDF, os restos de solvente, as tintas e os pigmentos, os restos de cola e a sucata de latas de alumínio. As alternativas propostas contemplam a aplicação de boas práticas operacionais, o reuso, a reciclagem interna e a fabricação de novos produtos. Essas proposições podem contribuir para melhorias da organização do sistema produtivo e do desempenho industrial, o que possibilita tornar o processo produtivo menos agressivo ao meio ambiente. Tem-se como perspectiva uma melhoria na imagem da empresa perante os seus colaboradores e clientes, bem como, a valorização das atividades que podem propiciar o aumento da lucratividade e reduzir o consumo de recursos naturais.

Palavras-chave: MDF (Medium Density Fiberboard). Tecnologias. Recursos naturais.

\section{Introdução}

O Brasil apresenta grande potencial de crescimento do setor moveleiro. Para favorecer esse crescimento, é necessário que as indústrias realizem investimentos em maquinários, adotem novas tecnologias no processo produtivo, qualifiquem a mão-de-obra, utilizem madeira seca de qualidade para capital de giro e realizem constantes investimentos na atividade [1].

A indústria de mobiliário faz parte dos setores tradicionais da economia, possuindo uma série de aspectos em comum, como o reduzido dinamismo tecnológico, a intensidade de mão-de-obra relativamente elevada e a grande utilização de materiais de origem vegetal e sintética [2].

No Brasil, essa atividade é caracterizada, principalmente, por micro e pequenas empresas, com predomínio de capital nacional. Existe um processo de produção diversificado conforme as matérias-primas empregadas nos produtos finais almejados, além de haver uma dependência de insumos, como plástico, metal, vidro, químico e têxtil [1], fornecidos por outras indústrias. A indústria moveleira emprega cerca de 300 mil trabalhadores diretos e gera 1,5 milhões de empregos indiretos em empresas que possuem entre 1 a 99 trabalhadores [3].

Por serem grandes geradoras de resíduos, é necessário que estes sejam gerenciados e destinados adequadamente. A variedade e a origem distinta dos materiais utilizados no processo industrial geram sobras do processo, como resíduos sólidos, emissões atmosféricas e efluentes líquidos [4].
Os resíduos da indústria moveleira podem deixar de ser um risco ao meio ambiente e passar a gerar lucro para a empresa. Para isso, é preciso olhar o resíduo não só como um problema, mas sim como um subproduto. Além disso, deveria se pensar em alternativas a serem adotadas para reutilizar esse material que é, muitas vezes, descartado de forma inadequada no meio ambiente [5].

Estudos afirmam que a utilização de novas tecnologias de minimização e reutilização de resíduos é fundamental para a sustentabilidade das indústrias moveleiras. Para isso, a eco eficiência pode ser uma alternativa que garanta melhorias na lucratividade e nos aspectos socioambientais das indústrias.

A eco eficiência refere-se a um modelo de produção e de consumo sustentável, proveniente da produção de bens e serviços que contribuem com a melhoria da qualidade de vida e para a valorização da reciclagem de resíduos.

A adoção da prevenção à poluição requer mudanças em processos e produtos, a fim de reduzir ou eliminar rejeitos na fonte, isto é, antes que eles sejam produzidos e lançados ao meio ambiente. Os rejeitos são produzidos, pois nenhum processo é $100 \%$ eficiente. Esses rejeitos devem ser captados, tratados e dispostos por meio de tecnologias de controle de poluição do tipo end-of-pipe. A prevenção da poluição aumenta a produtividade da empresa, proporcionando a redução de poluentes na fonte e evitando, dessa forma, o desperdício de recursos. Isso permite produzir mais bens de serviços com menos insumos. Os resultados esperados são a redução dos 
custos com materiais e energia, a economia na disposição final dos resíduos, a diminuição dos passivos ambientais, a melhoria geral das condições de trabalho e da imagem da empresa [6].

Com o crescimento da preocupação ambiental, as empresas passaram a adotar medidas voltadas à utilização racional de recursos, bem como a minimização de resíduos, já que a questão ambiental é, hoje, um requisito para tornar os produtos competitivos através do marketing verde [7].

Para propor medidas de prevenção à poluição, é necessário conhecer o processo produtivo, saber quais são as fontes poluentes, pois quanto maior for a perda de matériaprima no processo, maior será a produção de resíduos, maiores serão os custos para a empresa e maiores serão os prejuízos ao meio ambiente. A metodologia utilizada neste estudo relacionase à aplicação de prevenção à poluição e à priorização de resíduos. O estudo está embasado na proposta da EPA (Agência de Proteção Ambiental Norte-Americana). Por meio de levantamento, caracterização e quantificação dos resíduos produzidos pelo setor da empresa, a EPA possibilita definir os resíduos prioritários, que são aqueles com os quais a empresa deve se ocupar primeiramente [8].

Este artigo tem como objetivo apresentar de forma detalhada a aplicação de metodologias para implementação da prevenção à poluição (P2) em uma indústria moveleira, localizada na região oeste do estado do Paraná. Além disso, o artigo propõe alternativas para a minimização, o reuso, a reciclagem interna. Apresenta, também, boas práticas operacionais e novas tecnologias para lidar com os resíduos gerados no processo produtivo do setor de planos e MDF (Medium Density Fiberboard), com a finalidade de contribuir com o uso eficaz dos recursos naturais, melhorar a imagem da empresa, de seus produtos e, ao mesmo tempo, aumentar o seu desempenho econômico.

\section{Metodologia}

\subsection{Delimitação da área de estudo}

O estudo foi realizado em uma indústria moveleira, localizada no interior do estado do Paraná, mais especificamente na cidade de Medianeira. A empresa, caracterizada como de médio porte, possui área construída de $18.000 \mathrm{~m}^{2}$ e comporta, atualmente, aproximadamente 200 funcionários, distribuídos em seus diversos setores (administrativo, almoxarifado, manutenção, divisão de cadeiras, lixação, desdobramento, pintura, expedição, divisão de planos e MDF, embalagem e estofaria). Para o estudo, o setor delimitado foi o de planos e MDF. Este comporta a produção de bases de mesa, racks, aparadores, balcões, bancos e mezanino.

\subsection{Caracterização do processo produtivo}

A primeira etapa do processo produtivo da empresa é denominada de recepção e armazenamento. Nessa etapa, os insumos são recepcionados e armazenados em local específico, o MDF é estocado em uma área próxima às máquinas de corte e os outros insumos são acondicionados no almoxarifado, para a sua posterior utilização na produção de móveis. E, os resíduos gerados são as fitas plásticas oriundas da amarração do MDF. A empresa possui um funcionário específico que atua nessa função.

$\mathrm{Na}$ próxima etapa, o MDF é cortado e recebe uma cobertura com lâminas de madeira, conforme o produto a ser produzido. $\mathrm{O}$ processo de colagem das lâminas de madeira na chapa de MDF é realizado com o uso de uma resina de uréiaformol e o equipamento "rolo de cola". Posteriormente, a secagem das chapas ocorre em um equipamento-prensa a 80 ${ }^{\circ} \mathrm{C}$. Durante a lavagem do equipamento rolo de cola, ocorre a produção do efluente de resina uréia-formol, um passivo ambiental poluente considerado um biocida.

As chapas de MDF com revestimento de madeira, ou chapas de MDF cruas, são enviadas ao processo de usinagem para serem trabalhadas com maior detalhamento. Lá, recebem as medidas certas de acordo com o projeto final do produto. Nesse processo, maquinários para o pré-corte, a usinagem, a colagem de fita de borda são utilizados. Estes maquinários acarretam aspectos ambientais como consumo de energia elétrica, geração de ruídos e resíduos sólidos (maravalha, pó de MDF e restos de fita de borda).

Posteriormente ao corte, as peças recebem acabamentos, como a lixação e a pintura manual ou ultravioleta (UV). Durante a lixação, é possível observar a elevada geração de resíduos sólidos como lixas e abrasivos, pó de MDF, além do consumo de energia elétrica das lixadeiras e emissão de ruídos. $\mathrm{Na}$ pintura manual e ultravioleta, ocorre a utilização de variados compostos químicos como verniz, tingidor, thinner, primer, fundo, diluente, selante e, principalmente, o solvente. Como resíduos, observa-se a geração de efluentes de solvente misturados com esses materiais.

Na linha de pintura UV, é possível notar um alto consumo de energia elétrica. Após a pintura, a peça segue para a montagem/embalagem, onde são utilizados outros insumos como cola branca, parafusos, isomanta e isopor. Durante esse processo, ocorre a geração de resíduos sólidos tais como embalagens de cola, sacos plásticos e papelão.

Para a avaliação de aspectos e impactos ambientais, inicialmente, foi realizada a identificação dos aspectos ambientais das atividades da empresa no setor de MDF, a partir do fluxograma de processo, e posteriormente, foram 
determinados e avaliados os impactos ambientais associados a estes aspectos.

\subsection{Prevenção à poluição e minimização de resíduos}

No contexto de prevenção à poluição e minimização de resíduos na indústria moveleira, foi utilizada, de forma adaptada, a metodologia proposta pela Agência de Proteção Ambiental Norte-Americana (Environmental Protection Agency, EPA) [9], conforme ilustra a Figura 1.

\begin{tabular}{|c|c|}
\hline $\begin{array}{l}\text { PLANEJAMINTO E ORGANIZAÇÃO } \\
\text { Comprometimento da gerência (alta } \\
\text { administraçấo) } \\
\text { Estabelecer objetivos e metas } \\
\text { Formar equipe responsável pelo programa }\end{array}$ & \\
\hline $\begin{array}{c}\text { FASE DE AVALIAÇÃO } \\
\text { Coleta de dados no processo e instalações } \\
\text { Priorizacao e seleção dos alvos de avaliação } \\
\text { (residuos) } \\
\text { Revisão de dados }\end{array}$ & $\begin{array}{l}\text { Seleçào de novas } \\
\text { metas de avaliaçào e } \\
\text { reavaliação de } \\
\text { alternativas prévias }\end{array}$ \\
\hline $\begin{array}{c}\text { FASE DE ANÁLISE DE VIABILIDADE } \\
\text { Avaliaçào técnica } \\
\text { Avaliação econômica } \\
\text { Seleção de opçōes para implementação }\end{array}$ & Repeticào do processo \\
\hline $\begin{array}{c}\text { IMPLEMENTAÇÃO } \\
\text { Justificativa de projetos e obtençào de fundos } \\
\text { Instalação (equipamentos) } \\
\text { Implementaça (métodos procedimentos) } \\
\text { Avaliação de desempenho }\end{array}$ & \\
\hline
\end{tabular}

Figura 1 - Metodologia de minimização de resíduos [9].

$\mathrm{Na}$ etapa de planejamento, definiram-se os objetivos e as metas para a execução do trabalho, além da equipe responsável pela execução do programa. Já na fase de avaliação, realizou-se o levantamento de dados na linha de produção de MDF, com base no estudo de fluxograma do processo com balanço de massa, apresentado na Figura 2. Na fase de análise de viabilidade, avaliou-se o potencial da adoção de medidas para minimizar os resíduos por meio da aplicação da metodologia. A finalidade era definir os resíduos prioritários, para os quais se propôs as oportunidades de melhoria ambiental. Estas contemplaram a redução na fonte, mudanças no produto, controle na fonte-processo e reciclagem interna e externa.

Em relação à implementação, foram propostas medidas relacionadas à prevenção e à minimização de resíduos na indústria. $\mathrm{O}$ objetivo era permitir uma maior autonomia sobre a decisão de implementar as ações no tempo mais adequado, conforme a demanda dos processos produtivos e os recursos disponíveis.

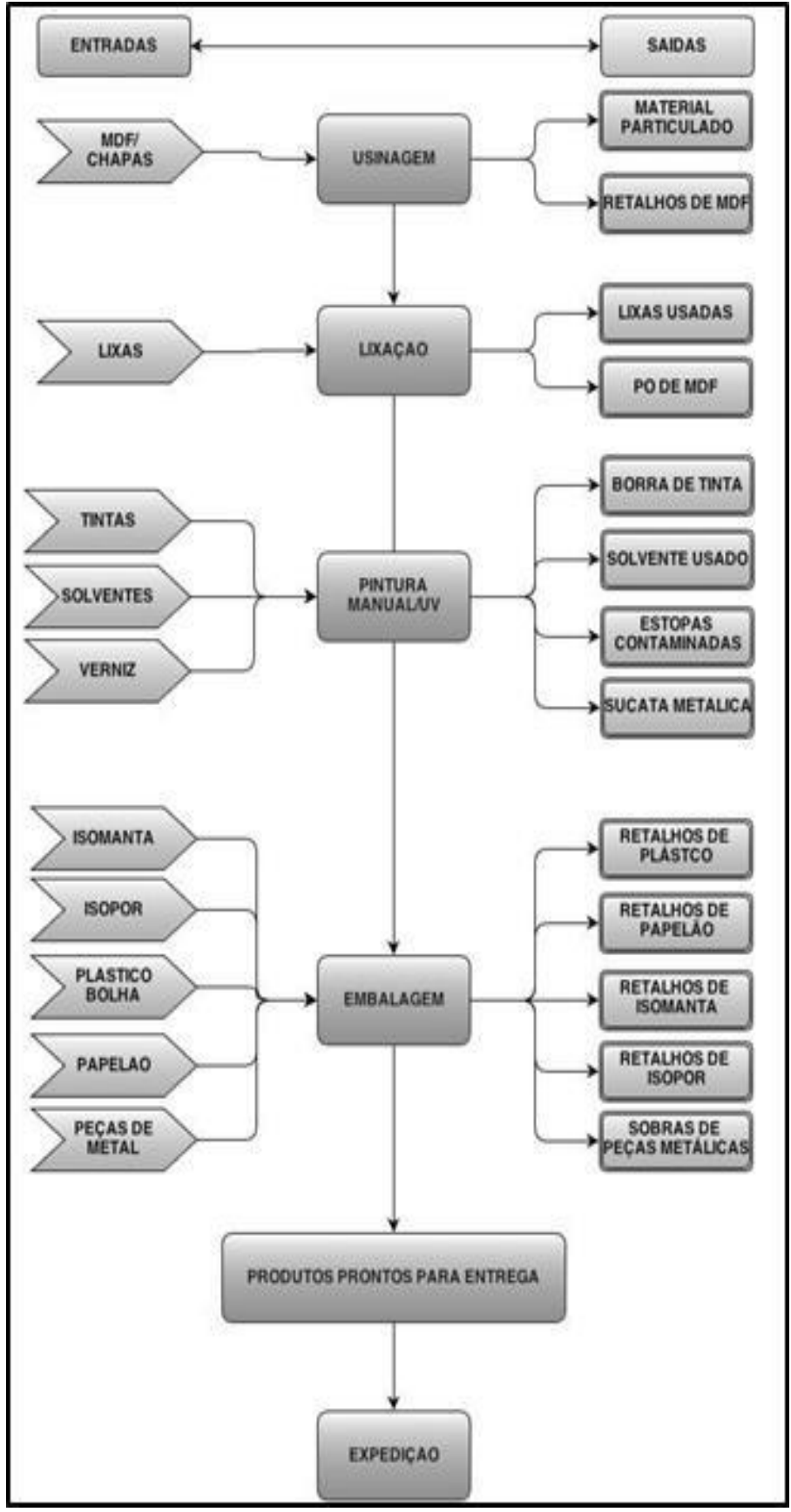

Figura 2 - Fluxograma do processo produtivo no setor de MDF. 


\subsection{Priorização de resíduos}

A priorização de resíduos foi uma adaptada da metodologia proposta pela Environmental Protection Agency [9], que apresenta os seguintes critérios para compor a tabela de priorização de resíduos:

A. Conformidade com a legislação;

B. Custos com tratamento e disposição final;

C. Riscos potenciais ao meio ambiente e à segurança;

D. Quantidade de resíduos gerada;

E. Propriedade de risco dos resíduos, conforme classificação do resíduo (incluindo a toxicidade, inflamabilidade, corrosividade e reatividade);

F. Potencial ou facilidade de minimização;

G. Potencial de recuperação de subprodutos valiosos;

H. Riscos para empregados - manuseio;

I. Orçamento disponível para o programa de avaliação e para projetos de minimização de resíduos;

J. Potencial para a remoção de gargalos na produção ou no tratamento de resíduos.

O Ecotime optou pela utilização de todos os critérios da EPA e, por meio de um brainstorming, estipulou a valoração de cada item (para A, B, C, D, E, F, G, H, I e J), de 1, 3 ou 9, e a atribuição de um peso para cada item (1, 2 ou 3), de acordo com a sua importância para a empresa [10]. Na quantificação dos impactos, foram utilizados os mesmos símbolos do QFD (Quality Function Deployment). Através do consenso, foi possível obter o número de prioridade com o uso da série $3^{\mathrm{n}}$.

$3^{0}=1$ : Probabilidade fraca ou inexistente;

$3^{1}=3$ : Probabilidade mediana;

$3^{2}=9$ : Alta probabilidade.

A metodologia foi adaptada segundo necessidades e aspirações da empresa.

Referente à conformidade com a legislação (A), equipe verificou os requisitos legais acerca da manipulação e da disposição dos resíduos. Para isso, a valoração foi estipulada em:

1 PONTO: Manipulação e disposição do resíduo atendem aos requisitos legais;

3 PONTOS: Manipulação e disposição conformes, mas com possibilidade de melhorias;

9 PONTOS: Manipulação e disposição não atendem aos requisitos legais.

Em relação à empresa em foco, atribuiu-se o peso 1 para a valoração, visto que o atendimento à legislação não foi uma prioridade apresentada por ela.
Referente ao item que aborda os custos com tratamento e disposição final dos resíduos (B), foram adotados a geração, o transporte e o fator de impacto com base nas despesas provenientes da destinação dos resíduos. Para este requisito, estipulou-se a valoração em:

1 PONTO: Custos menores que R\$ 2.000,00 ao ano;

3 PONTOS: Custos entre R \$ 2.000,00 e R \$ 5.000,00 ao ano;

9 PONTOS: Custos acima de R \$ 5.000,00 ao ano.

$\mathrm{Na}$ valoração desse critério, foi atribuído peso $3 \mathrm{e}$ priorização dos resíduos que geram mais custos à empresa.

O critério relacionado aos riscos potenciais ao meio ambiente e à segurança (C) somente foi empregado para resíduos de Classe I e de Classe IIA, classificados de acordo com a NBR 10.004/2004 [11], por referir-se aos riscos de saúde e à segurança dos funcionários. Estes foram valorados com peso 3. Os resíduos de Classe II B (inertes) não foram valorados. Para a valoração dos resíduos de Classe I (perigosos) e de Classe IIA (não-inertes), foram definidos:

1 PONTO: Resíduo não entra em contato com funcionários em nenhuma etapa de sua manipulação e acondicionamento;

3 PONTOS: Possibilidade de o funcionário entrar em contato com o resíduo;

9 PONTOS: Necessidade de contato direto do funcionário com o resíduo.

A quantidade de resíduo gerada (D) foi monitorada diariamente por funcionários da empresa, que quantificaram os materiais. Para a valoração desse item, foram estabelecidos:

1 PONTO: Até 1 t/ano de resíduo;

3 PONTOS: De 1 t até 3 t/ano de resíduo;

9 PONTOS: Mais de 3 t/ano de resíduo.

O peso atribuído à valoração desse item foi 3 , com prioridade aos resíduos gerados em maiores quantidades.

A classificação dos resíduos (E) foi realizada de acordo com a NBR 10.004/2004 [11], a qual dispõe a identificação e a classificação dos resíduos sólidos. Para a valoração, definiu-se:

1 PONTO: Resíduo Classe IIB;

3 PONTOS: Resíduo Classe IIA;

9 PONTOS: Resíduo Classe I.

Para a valoração deste item foi determinado o peso 2, conforme a necessidade da empresa sobre a geração de resíduos.

Para potencial ou facilidade de minimização (F), considerou-se que os itens mais fáceis de serem implementados 
eram aqueles que não geravam custos elevados à empresa. Nesse sentido, a valoração ocorreu da seguinte maneira:

1 PONTO: Investimento para minimizar o resíduo, com tempo de retorno de 1 a 2 anos;

3 PONTOS: Investimento para minimizar o resíduo, com tempo de retorno menor que 1 ano;

9 PONTOS: Não há necessidade de investimentos.

Devido à importância do item apresentado, foi lhe atribuído peso 3, já que existiam oportunidades na empresa para minimizar a geração de resíduos.

Para o item potencial de recuperação de subprodutos valiosos $(\mathrm{G})$, consideraram-se as possibilidades de reaproveitamento e de reciclagem de solventes, com a finalidade de otimizá-los. Além disso, para reaproveitar parte do efluente a partir do beneficiamento de uma quantidade de resíduo que retorne como matéria-prima para o processo, o que garante a redução de custos da empresa. Atribuiu-se:

1 PONTO: Resíduos passíveis de serem reaproveitados com medidas simples de tratamento;

3 PONTOS: Resíduos passíveis de serem reaproveitados sem tratamento;

9 PONTOS: Resíduos que são desperdiçados pelo mau uso e por destinação incorreta.

O peso atribuído a este item foi 2, pois levou-se em consideração a melhoria da gestão de resíduos da empresa.

Em relação aos riscos aos empregados $(\mathrm{H})$, foram considerados perigos decorrentes do manuseio dos resíduos gerados. Os critérios para a valoração foram:

1 PONTO: Não apresenta risco decorrente do seu manuseio;

3 PONTOS: Pode apresentar risco decorrente do seu manuseio;

9 PONTOS: Altíssimo risco decorrente do seu manuseio.

Com base nesse critério, atribuiu-se o peso de valor 1 , visto que a empresa possui a CIPA (Comissão Interna de Prevenção de Acidentes), que está direcionada aos aspectos prevencionistas nas relações empregador-empregado.

Referente ao orçamento disponível para o programa de avaliação e para projetos de minimização de resíduos (I), valores mais elevados foram proporcionais à quantidade de recursos para sua minimização. A valoração ocorreu da seguinte maneira:

1 PONTO: Necessita de investimentos superiores a US\$ 500,00 para a minimização dos resíduos;

3 PONTOS: Necessita de investimentos inferiores a US\$ 500,00 para a minimização dos resíduos;
9 PONTOS: Não necessita de recursos para a minimização dos resíduos.

Em relação a esse critério, o peso atribuído foi de valor 1, pois não apresenta relevância para a empresa.

Sobre o potencial para a remoção de gargalos na produção ou no tratamento de resíduos $(\mathrm{J})$, foram avaliados os possíveis benefícios decorrentes da minimização de resíduos na fonte da indústria. Para a valoração, definiu-se:

1 PONTO: Remoção de gargalos demanda recursos financeiros e adoção de boas práticas operacionais;

3 PONTOS: Remoção de gargalos irá demandar recursos financeiros para aquisição de equipamentos que contribuam para um melhor rendimento do processo produtivo;

9 PONTOS: Remoção de gargalos é possível com a adoção de boas práticas operacionais.

Para esse critério, o peso atribuído foi de valor 2, visto que a empresa admite a busca da melhoria contínua de seus processos.

A definição do Número de Prioridade (N.P.) foi orientada de acordo com a metodologia adaptada [10], na qual o número de prioridade é a somatória dos dez itens anteriores (A, B, C, D, E, F, G, H, I e J), que foram valorados a partir de um brainstorming. Estes podem apresentar valores de 1, 3 ou 9 multiplicados pelos seus respectivos pesos $\left(\mathrm{P}_{\mathrm{A}}, \mathrm{P}_{\mathrm{B}}, \mathrm{P}_{\mathrm{C}}, \mathrm{P}_{\mathrm{D}}, \mathrm{P}_{\mathrm{F}}\right.$, $\mathrm{P}_{\mathrm{G}}, \mathrm{P}_{\mathrm{H}}, \mathrm{P}_{\mathrm{I}}, \mathrm{P}_{\mathrm{J}}$ ), de 1,2 ou 3 , conforme o interesse da empresa.

\section{N.P. $=A \times P_{A}+B \times P_{B}+C \times P_{C}+D \times P_{D}+E \times P_{E}+F \times P_{F}+G \times P_{G}+H \times P_{H}+I \times P_{I}+J \times P_{J}$}

Os resíduos com maiores N.P. são considerados os de maior prioridade para estudo. $\mathrm{O}$ item referente à quantidade de resíduo gerada (D) foi utilizado como critério para desempate.

O cálculo de N.P. de um dos resíduos, como o pó e a serragem de MDF, produzidos na indústria estudada, foi obtido a partir da substituição da valoração e do peso de cada item. Seguindo-se a equação citada, obteve-se o seguinte N.P. para este resíduo:

\section{N.P. $=(3 \times 1)+(9 \times 3)+(9 \times 3)+(9 \times 3)+(3 \times 2)+(1 \times 3)+(1 \times 2)+(3 \times 1)+(3 \times 1)+(1 \times 2)$}

$$
\text { N.P. }=103
$$

\subsection{Oportunidades para a minimização de resíduos}

A caracterização das oportunidades para a aplicação da prevenção à poluição e à minimização de resíduos apresenta como base o fluxograma adaptado da EPA [9]. Este estudo sugere as seguintes etapas, conforme Figura 3: 


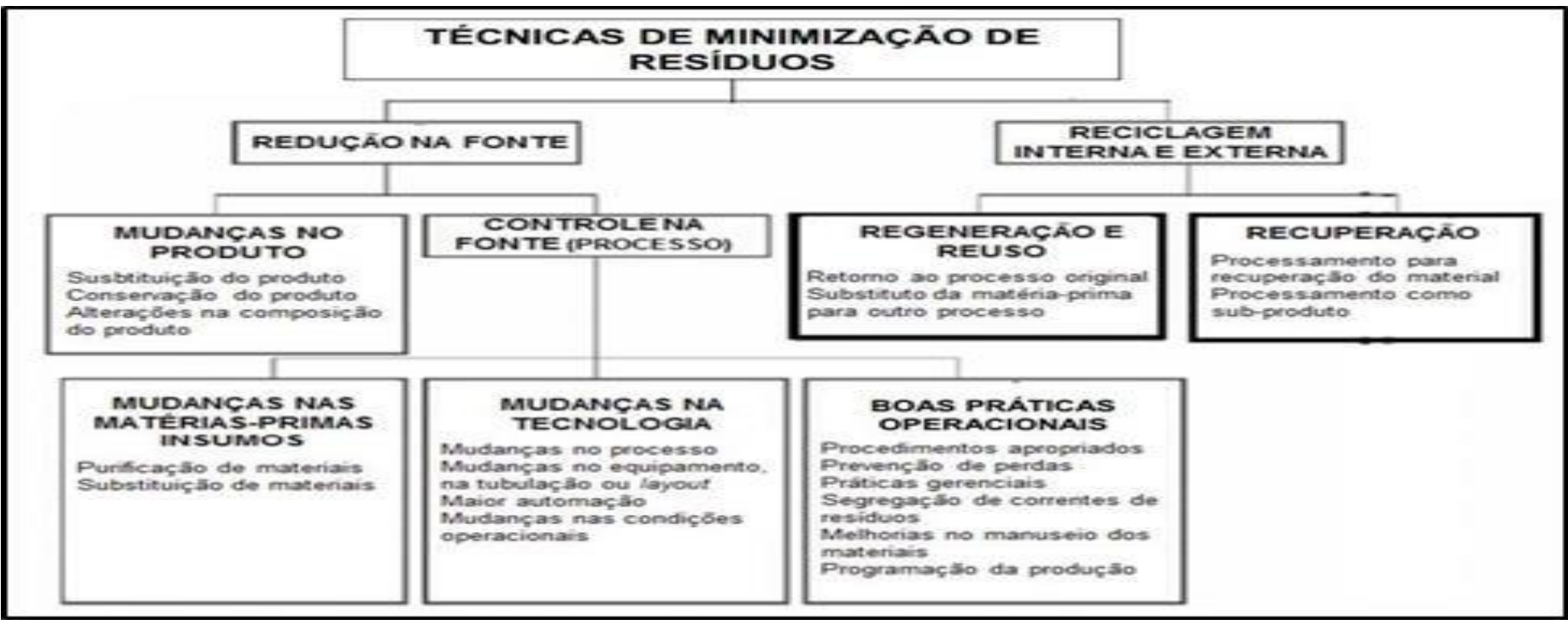

Figura 3 - Técnicas de minimização de resíduos.

Fonte: Adaptado de EPA [9].

A maior prioridade é atribuída à redução na fonte. A redução pode ocorrer por meio de modificações em produtos $\mathrm{e}$ em processos, com base na priorização dos resíduos e com o controle da fonte geradora. Podem, também, ser realizadas mudanças nas tecnologias empregadas no processamento industrial, além de reciclagem interna e externa. A Tabela 1 apresenta os resíduos e os insumos estudados e a quantidade gerada no setor produtivo de MDF.

Tabela 1. Levantamento de resíduos e de custos do setor de MDF.

\begin{tabular}{|c|c|c|c|}
\hline Resíduos & Custos & Quantidade & Classe \\
\hline Resíduos de plástico & $\mathrm{US}^{1} 342.53$ & 2.456 (kg/ano) & II B \\
\hline Resíduos de papel e papelão & US\$ 620.43 & 8.897 (kg/ano) & II B \\
\hline Sucata de latas de alumínio & US\$ 331.00 & 4.748 (kg/ano) & II B \\
\hline Restos de solvente, tintas e pigmentos & US\$ -1.557.41 & 6.700 (litros/ano) & I \\
\hline Abrasivos e lixas de papel & US\$ - 627.61 & 3.000 (kg/ano) & II A \\
\hline Isomanta/Isopor & US\$ - 418.41 & $2.000(\mathrm{~kg} / \mathrm{ano})$ & II B \\
\hline EPI usado & US\$ - 219.20 & $1.000(\mathrm{~kg} / \mathrm{ano})$ & II A \\
\hline Fitilho de ráfia & US\$ - 334.72 & $1.600(\mathrm{~kg} / \mathrm{ano})$ & II B \\
\hline Efluente resina uréia-formol & US\$ - 2324.50 & 1.0000 (litros/ano) & I \\
\hline Estopas usadas & US\$ - 209.20 & 1.000 (kg/ano) & II A \\
\hline Pó e serragem de MDF & US\$ - 2789.40 & $2.0000(\mathrm{~kg} / \mathrm{ano})$ & II A \\
\hline Retalhos de madeira densificada (MDF) & US\$ - 232.45 & $1.000(\mathrm{~kg} / \mathrm{ano})$ & II A \\
\hline Fita de borda PVC & US\$ - 69.73 & $500(\mathrm{~kg} / \mathrm{ano})$ & II B \\
\hline Restos de cola & US\$ - 209.20 & $1.000(\mathrm{~kg} / \mathrm{ano})$ & II A \\
\hline \multicolumn{4}{|l|}{ Insumos } \\
\hline Água & US\$ - 3719.20 & $480.000\left(\mathrm{~m}^{3} / \mathrm{ano}\right)$ & \\
\hline Energia Elétrica & US\$ - 111576.01 & 1.669 .554 (kw/ano) & \\
\hline
\end{tabular}

\footnotetext{
${ }^{1}$ Cotação consultada no dia 17 jun. 2013, um US\$ 1.00 equivale a R\$ 2,151;
} 


\section{Resultados e discussões}

Entre os resíduos analisados, foram atribuídos critérios para priorização e proposição de medidas aos dez resíduos prioritários, conforme a ordem de classificação a ser observada na Tabela 2. Para a minimização na fonte, estabeleceu-se como Tabela 2. Resíduos na ordem de priorização de acordo com N.P.

\begin{tabular}{ccccccccccccc}
\hline Ordem & Resíduos & A & B & C & D & $\mathbf{E}$ & $\mathbf{F}$ & $\mathbf{G}$ & $\mathbf{H}$ & $\mathbf{I}$ & $\mathbf{J}$ & $\mathbf{N P}$ \\
\hline $1^{\circ}$ & Efluente resina uréia-formol & 3 & 27 & 27 & 27 & 18 & 3 & 2 & 1 & 1 & 2 & 111 \\
$2^{\circ}$ & Pó e serragem de MDF & 3 & 27 & 27 & 27 & 6 & 3 & 2 & 3 & 3 & 2 & 103 \\
$3^{\circ}$ & Restos de solvente, tintas e pigmentos & 3 & 9 & 9 & 27 & 18 & 3 & 6 & 9 & 1 & 6 & 91 \\
$4^{\circ}$ & Restos de colas & 1 & 3 & 9 & 3 & 6 & 27 & 6 & 9 & 9 & 18 & 91 \\
$5^{\circ}$ & Sucata de latas de alumínio & 1 & 3 & 9 & 27 & 2 & 27 & 6 & 1 & 3 & 2 & 81 \\
$6^{\circ}$ & Abrasivos e lixas de papel & 1 & 3 & 27 & 9 & 6 & 3 & 6 & 3 & 3 & 6 & 67 \\
$7^{\circ}$ & Isomanta/Isopor & 1 & 3 & 3 & 3 & 2 & 9 & 18 & 1 & 9 & 18 & 67 \\
$8^{\circ}$ & Energia Elétrica & 1 & 3 & 3 & 3 & 2 & 27 & 2 & 3 & 3 & 18 & 66 \\
$9^{\circ}$ & Resíduos de plástico & 1 & 3 & 3 & 9 & 2 & 27 & 6 & 1 & 9 & 2 & 63 \\
$10^{\circ}$ & Água & 1 & 3 & 3 & 3 & 2 & 27 & 2 & 1 & 3 & 18 & 63 \\
$11^{\circ}$ & Estopas usadas & 1 & 3 & 9 & 3 & 6 & 27 & 6 & 1 & 3 & 2 & 61 \\
$12^{\circ}$ & Resíduos de papel e papelão & 1 & 3 & 3 & 27 & 2 & 3 & 6 & 1 & 9 & 2 & 57 \\
$13^{\circ}$ & EPI usado & 1 & 3 & 3 & 3 & 6 & 3 & 6 & 1 & 9 & 2 & 37 \\
$14^{\circ}$ & Retalhos de Madeira Densificada (MDF) & 3 & 3 & 9 & 3 & 6 & 3 & 2 & 3 & 3 & 2 & 37 \\
$15^{\circ}$ & Fitilho de ráfia & 3 & 3 & 3 & 3 & 2 & 3 & 2 & 1 & 9 & 6 & 35 \\
$16^{\circ}$ & Fita de borda PVC & 3 & 3 & 3 & 3 & 2 & 3 & 2 & 3 & 3 & 2 & 27 \\
\hline
\end{tabular}

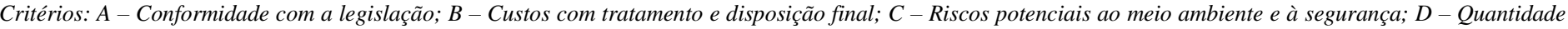

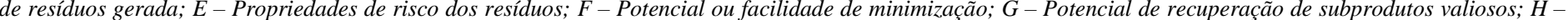

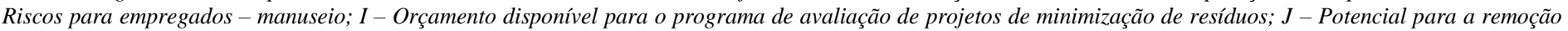
de gargalos na produção ou no tratamento de resíduos.

\subsection{Proposição das melhorias conforme os resíduos prioritários}

\subsubsection{Efluente resina uréia-formol}

Observou-se a necessidade de reduzir os custos com a disposição final deste resíduo, visto que o mesmo é terceirizado. Nesse sentido, a técnica de minimização proposta é a reciclagem interna. É interessante estudar a possibilidade de utilizar o efluente como substância agregadora na fabricação de chapas recicladas de MDF. Com essa proposição, o resíduo pode ser reaproveitado em um novo processo produtivo, cuja matériaprima também será um resíduo (aparas de pó e serragem de $\mathrm{MDF}$ ), originado como segundo item da lista de prioridades.

O efluente é gerado pelo processo de limpeza do equipamento "rolo de cola". Na Figura 4 é possível observar que a limpeza é realizada com uma mangueira com vazão simples, visando à minimização do consumo de água neste processo. É medida o controle na fonte. Não sendo possível a redução na fonte, estabeleceram-se medidas para reciclagem interna ou externa. 


\subsubsection{Retalhos, pó e serragem de $M D F$}

Para realizar a reciclagem interna com o reuso de retalhos de MDF, propôs-se a separação dos resíduos de acordo com as suas dimensões e a utilização para outros fins. Isso foi possível porque a empresa utiliza maquinários para corte que possuem controle numérico computadorizado na produção. As formas dos retalhos gerados são semelhantes. Logo, torna-se possível o reuso desses retalhos descartados como resíduos (Figura 5). Propõe-se a produção de novos produtos a partir desses retalhos, o que evita o seu desperdício.

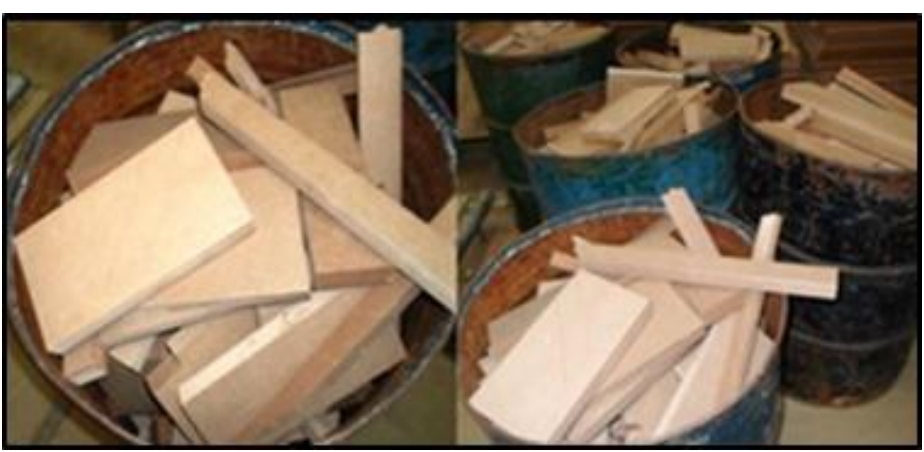

Figura 5 - Retalhos de MDF não aproveitados.

Uma das dificuldades relacionadas ao uso de retalhos de MDF é que, por se tratarem de pedaços e não de chapas inteiras, eles podem se tornar quebradiços e pouco resistentes, dependendo do lado em que são trabalhados. Os retalhos podem, no entanto, ganhar reforço com a fita de borda ou com adesivos sintéticos. Uma forma de reutilização destes materiais é o uso dos pedaços de MDF para produzir bases de mesas de centro com tampo de vidro, como a apresentada na Figura 6 [13].

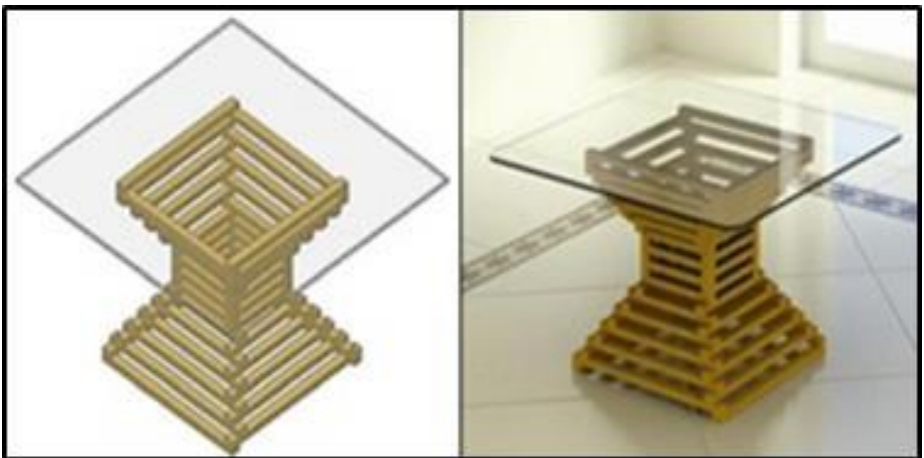

Figura 6 - Mesa de centro com base em formato de ampulheta [15].

Para produzir essa mesa de centro, primeiramente, é necessário realizar o corte, em uma base plana. Com o auxílio do esquadro, são alinhadas as primeiras peças. Já com o uso de cola branca, as peças são sobrepostas e coladas pelas extremidades. Depois de montada a base, retira-se o excesso de cola e realiza-se a limpeza da peça. O tampo utilizado na peça, como mostra a Figura 6, é um vidro temperado com peso de $11,25 \mathrm{~kg}$, incolor, com acabamento lapidado e canto tipo moeda, com polimento e brilho natural. Dessa maneira, é possível reutilizar o resíduo e transformá-lo em matéria-prima na fabricação de um novo produto [13].

Outra alternativa que agrega valor a este subproduto é a reciclagem interna, por meio da implantação de um equipamento tipo prensa para a fabricação de chapas recicladas de MDF. Uma maneira de aproveitar as aparas de MDF de $15 \mathrm{~mm}$ geradas na produção é empregar uma prensa de sobras de MDF na fabricação de chapas recicladas. Assim, também, se obtém um novo produto, conforme ilustrado na Figura 7 [7].

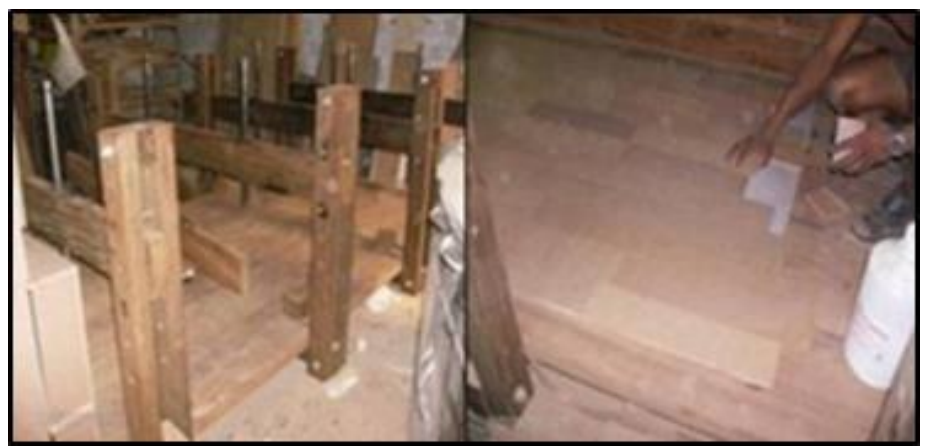

Figura 7 - Fabricação de novas chapas com o uso da prensa [7].

O pó e a serragem de MDF são provenientes dos equipamentos de corte, usinagem, furação. Estes estão interligados através de tubulação exaustora aos filtros de manga, que compõem o sistema de tratamento de emissões atmosféricas da empresa. Os filtros ficam retidos em um silo até o momento da disposição final.

São poucas as propostas voltadas para o reaproveitamento da serragem e do pó. Os resíduos de MDF não podem ser utilizados como forração de granjas, adubo, fonte de energia térmica, nem mesmo ser incinerados sem controle. Pois, apresentam em sua composição substâncias tóxicas originadas das resinas adesivas, que geralmente são à base de uréiaformaldeído, de fenol-formaldeído, melamina-formaldeído ou uréia-formaldeído-melamina [14].

Os painéis também necessitam do uso de outras substâncias como os fungicidas e/ou inseticidas, a parafina para impermeabilizar o material, os adesivos, a cola e os solventes. $\mathrm{O}$ uso dessas substâncias tóxicas faz com que seja necessário adotar medidas adequadas à destinação desses resíduos [15].

Uma das proposições para a serragem é a reciclagem interna com a elaboração de um novo produto, uma chapa reciclada de MDF.

O MDF é produzido originalmente a partir da redução de madeiras a fibras por processos termomecânicos, uma sequência de ações de temperatura e pressão. Essas partículas são aglomeradas com resinas sintéticas (uréia-formaldeído, ou fenolformaldeído) para que se unam formando os painéis de média densidade [16]. 
Com base nisso, sugere-se a viabilização de um equipamento que faça a mistura do efluente de resina uréiaformol ao pó e às aparas de MDF. E, que se produza uma massa passível de moldagem, onde possa ser aplicada uma prensa quente para incorporação desse material. A placa produzida poderá ser utilizada no reforço de encostos de cadeiras, doações aos artesões do município, entre outros usos. É possível aplicar a serragem na produção industrial de painéis de MDF. Em estudos realizados com o uso de três proporções de serragem $(10,20$ e $30 \%$ ), foi constado que os painéis fabricados com o uso de $10 \%$ de serragem apresentam um produto de qualidade semelhante ao MDF obtido da madeira roliça. Porém, com o aumento da serragem ocorreu a redução das propriedades mecânicas, o que afetou a estabilidade dimensional dos painéis [17].

Entre as propostas de destino adequado desses resíduos provenientes de painéis de MDF, está a confecção de tijolos de solo-cimento, que são reforçados com o uso dessa serragem. $\mathrm{Na}$ fabricação desse tipo de tijolo, utiliza-se como matéria-prima solo, cimento e fibras de resíduos de madeira. Entre as vantagens de seu uso, pode-se destacar a possibilidade de confecção de componentes leves, com bom desempenho mecânico, capazes de controlar a propagação de fissuras, além do destino adequado a esse resíduo [18].

Outra proposta para a destinação correta do resíduo é a utilização da produção de compósitos de plástico-madeira, materiais recicláveis de boa qualidade e com grande aplicabilidade [19].

Os "compósitos são materiais formados por uma fase contínua polimérica (matriz), reforçados por uma fase descontínua (fibras)". Seu uso é interessante devido às características de não rachar, não empenar e, praticamente, não necessitar de manutenção. Este composto pode ser usinado, serrado, parafusado e aplainado em máquinas da indústria moveleira, como ocorre com a própria madeira [19].

\subsubsection{Solventes, tintas, pigmentos e restos de cola}

Como proposições para o controle na fonte por meio da aplicação de boas práticas operacionais para a otimização do consumo de tintas, sugere-se a análise do sistema de ar comprimido utilizado nas pistolas de pintura manual. A finalidade é regular a pressão das pistolas utilizadas nas cabines de pintura de acordo com as informações disponibilizadas pelo fabricante do equipamento e pelo fabricante da tinta [20].

Uma proposta para minimização na fonte, devido ao desperdício de tintas, solventes, cola de cianoacrilato, cola de madeira e cola branca, é o emprego de um móvel, conforme modelo (Figura 8), para realizar o escoamento do resto do solvente que fica retido na embalagem após o uso [7]. Visto que a retirada de novos insumos é realizada por planilhas de controle do almoxarifado, que solicita aos funcionários a devolutiva da embalagem usada para o fornecimento de uma nova, este escoamento poderá ser realizado imediatamente após a entrega das embalagens usadas dentro do setor de almoxarifado. Lá, será inserido o móvel com o objetivo de aproveitar os produtos que ficam retidos no fundo da embalagem. Após esse procedimento, será possível reduzir o desperdício dos produtos citados.

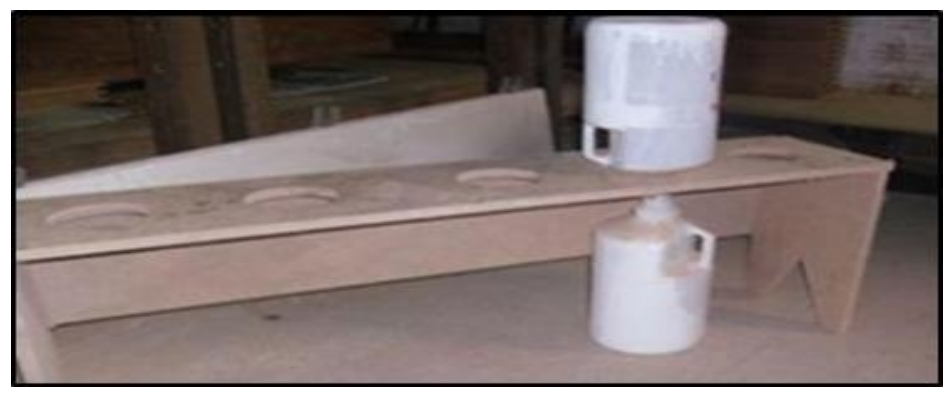

Figura 8 - Móvel utilizado para aproveitar restos de produtos [7].

Ao observar a periculosidade e os danos ambientais causados pela destinação incorreta desses produtos químicos, uma atenção especial deve ser dada aos resíduos provenientes da linha de pintura.

A linha de pintura UV de uma indústria moveleira compreende um processo contínuo. Observa-se na Figura 9 uma máquina com vários segmentos em rolos que aplicam fundos, vernizes e tingimentos sobre painéis calibrados. O processo de pintura baseia-se, primeiramente, no lixamento da peça. Posteriormente, é feito o seu amassamento para corrigir os defeitos. Isso é feito com a aplicação de um fundo ou selador que sela os poros, nivela a superfície e promove, se necessário, seu lixamento intermediário, que deixará a superfície mais nivelada. Logo, nessa fase, o substrato é submetido a camadas de primer UV, que compreende um fundo com pigmento similar ao design que se pretende obter no MDF. Para finalizar o processo, chegase à etapa de acabamento, em que ocorre a aplicação de verniz brilhante ou fosco [21].

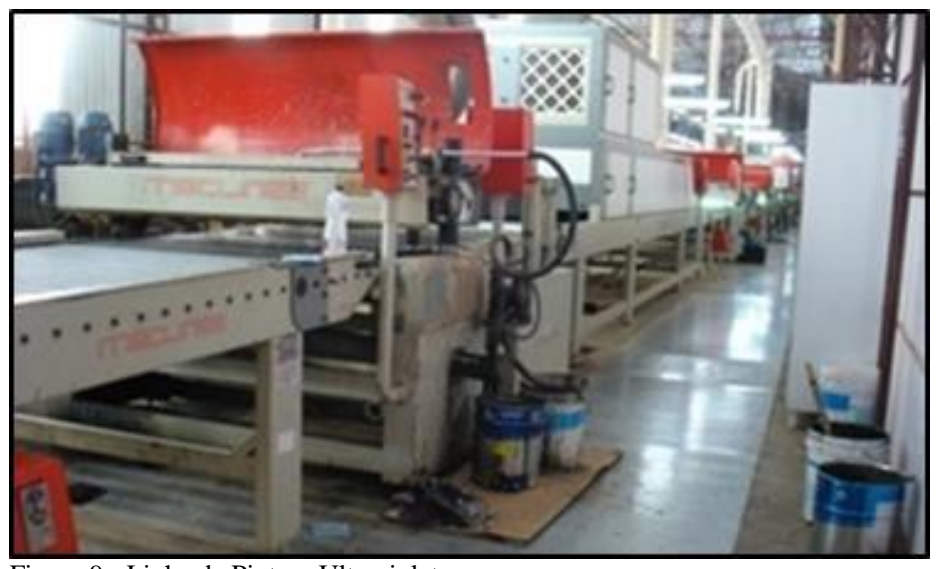

Figura 9 - Linha de Pintura Ultravioleta. 
Por meio de boas práticas operacionais, é possível orientar os funcionários quanto ao controle e à segregação dos distintos efluentes que contêm solventes em seu estado puro e misturados com água.

Os solventes são resíduos valiosos que podem ser recuperados por meio de uma destilação simples, com a adaptação de um equipamento específico ao processo produtivo. A destilação simples é o processo mais empregado na recuperação de solventes. Por meio de processos de destilação simples ocorre a separação do solvente e dos contaminantes. Com isso, o solvente, praticamente, volta à suas condições iniciais. Para isso, o processo de filtragem deve preceder a destilação [22]. A filtragem é utilizada para promover a retenção de sólidos e para contribuir no aumento da vida útil do equipamento de destilação, pois reduz a quantidade de sólidos impregnados no aparelho, no final do processo [21].

Para a recuperação de subprodutos por meio de reciclagem interna, sugere-se a implantação de um sistema de recuperação do solvente, conforme apresentado pela Figura 10.

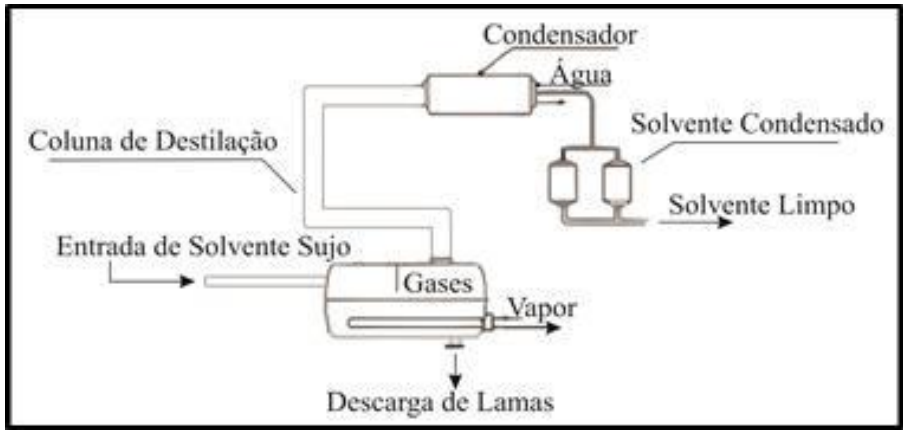

Figura 10. Sistema de destilação simples alimentado por vapor [21].

A proposição dessa alternativa está embasada em estudos que afirmam que as empresas, ao optarem pela reciclagem do resíduo de tinta, reduzem cerca de $50 \%$ nos custos com a compra de solventes [21].

\subsubsection{Aplicação de boas práticas operacionais}

Com vistas à redução da geração de sucata de latas de alumínio, é possível verificar a necessidade de um estudo para a substituição das embalagens pequenas por embalagens maiores. Estas reduzirão o volume e, consequentemente, a redução dos custos na compra dos insumos.

É necessário, também, planejar o uso da isomanta e do isopor e realizar o reaproveitamento interno. Evita-se, assim, o descarte do material. Com medidas simples de orientação aos funcionários, é possível reduzir o desperdício, pois a isomanta é amplamente utilizada desde a linha de pintura UV, quando se garante a proteção das peças contra riscos e atrito. Isso vale, até mesmo, para embalagens que fazem parte da camada de materiais que garantem a integridade do produto, visto que a empresa exporta para vários estados do Brasil.
Os abrasivos e lixas de papel podem ser minimizados com mudanças na forma de uso das folhas de lixas. Observou-se que as funcionárias da lixação dobram em quatro partes a lixa e utilizam apenas duas partes. Logo, aquelas que ficam nas partes posteriores são desperdiçadas, ou seja, metade da lixa que está no interior da dobra. Para este problema, sugere-se o corte na metade da folha da lixa, que possui $28 \mathrm{~cm}$ por $23 \mathrm{~cm}$, para que se possa utilizar ambos os lados da folha. Em relação às espumas abrasivas em rolo, orienta-se que sejam cortadas em tamanhos padronizados, conforme a necessidade do uso. Além disso, sugere-se que esse controle seja realizado pelo setor de almoxarifado, pois os funcionários, ao realizarem o corte por conta própria, acabam desperdiçando material por não possuírem a orientação sobre o tamanho adequado para o uso.

Entre as boas práticas operacionais para a indústria no que se refere à redução do gasto de energia elétrica, sugere-se a organização da produção por meio do uso de planilhas de controle. Estas devem viabilizar as quantidades a serem produzidas a cada dia, com a finalidade de reduzir a ociosidade dos equipamentos que permanecem ligados sem produzir. Um exemplo a ser mencionado é o maquinário da linha de pintura $\mathrm{UV}$, que permanece em funcionamento durante todo horário comercial, sem paradas, apesar de a máquina nem sempre estar em produção. Com programação, organização do sistema produtivo e acumulação de peças para realização da pintura em um único turno, estaria se economizando energia elétrica.

\section{Considerações finais}

Este trabalho evidencia a importância da aplicação das metodologias discutidas na identificação dos principais resíduos produzidos na indústria. Consequentemente, isso permite propor à empresa moveleira medidas voltadas à redução de desperdícios de recursos e geração de resíduos, eliminando, assim, alguns passivos ambientais.

Com o uso de metodologias específicas para prevenção à poluição e para a priorização de resíduos, foi possível identificar os resíduos prioritários no setor de divisão de planos e MDF e propor sugestões voltadas à redução da geração, à minimização de resíduos na fonte e à reciclagem interna. Além disso, sugeriuse a adoção de práticas operacionais que podem contribuir para as melhorias da organização do sistema produtivo e do desempenho industrial.

A quantidade de opções elaboradas indica a existência de um potencial latu de opções para a minimização de resíduos gerados no setor de planos e MDF da indústria.

Grande parte do desperdício de matérias-primas e insumos ocorre durante o processo produtivo. Este pode ser caracterizado como falta de conhecimento por parte da própria indústria sobre os seus aspectos e impactos ambientais. Além de haver falhas organizacionais, como a ausência de uma fiscalização e/ou 
controle da gerência para um uso racional dos recursos, observase a ausência de conhecimento e atitude ambiental, como o zelo pelos recursos ou a adoção de critérios para se evitar desperdícios e geração de resíduos por parte dos funcionários.

É possível concluir que estes fatores acarretam desperdícios significativos de materiais, geração de resíduos sólidos e efluentes, redução da produtividade e aumento dos riscos ao meio ambiente e aos funcionários. Por fim, evidencia-se a aplicação das metodologias de prevenção à poluição e de priorização de resíduos como ferramentas benéficas para a resolução dos problemas de gerenciamento ambiental das indústrias.

\section{Agradecimentos}

Agradecemos à Marivane Turim Koschevic, por todo apoio no desenvolvimento deste trabalho.

\section{METHODS AND MEASURES FOR REDUCING WASTE ON A FURNITURE INDUSTRY}

ABSTRACT: This study was carried out in a furniture industry located in the West of the state of Paraná, and presents the application of methodologies for prioritization and minimization of waste proposed by the EPA, under the approach of control at the source and internal recycling. Priority wastes were identified according to the Priority Number (N.P.), and selected the top ten items to the proposition of measures among them, ureaformaldehyde resin effluent, dust and MDF sawdust, the remains of solvent, inks and pigments, the remains of adhesives and scrap of aluminum cans. The proposed alternatives include the application of good operating practices, reuse, internal recycling, and the manufacture of new products. These propositions aim to contribute to improvements in the organization of the production system and the industrial performance, which enables the production process to become less aggressive to the environment, from the perspective of improving the image of the company for its employees and customers, as well as value these activities which may provide increased profitability and reduce the consumption of natural resources.

Keywords: MDF (Medium Density Fiberboard). Technologies. Natural resources.

\section{Referências}

[1] SOUZA, L. S. de; SOUZA, N. de J. de; Indústria moveleira de Boa Vista: estrutura e potencialidades. Análise. Porto Alegre, v.20, n.2, p. 120-136, jul./dez. 2009.

[2] ROSA, S. E. S.da.; CORREA, A. R. ; Lemos, M. L. F. ; BARROSO, D. V.O Setor de Móveis na Atualidade: uma Análise Preliminar. BNDES Setorial, Rio de Janeiro, n. 25, p. 65-106, mar. 2007.
[3] PRADO, L. L. A questão socioambientalnas empresas moveleiras do PólodeVotuporanga- SP.Revista Brasileira de Gestão e Desenvolvimento Regional.Taubaté, v. 7, n. 3, p. 27-51, set./dez. 2011

[4] ANTONIO, M. Avaliação do material particulado gerado em uma indústria moveleira e dimensionamento de um filtro de manga. 2010. $74 \mathrm{f}$. Trabalho de conclusão de curso. (Curso de Engenharia Ambiental) - Universidade de Passo Fundo, Passo Fundo, 2010

[5] LIMA, E. G. de; SILVA, D. A. da. Resíduos gerados em indústrias de móveis de madeira situadas no pólo moveleiro de Arapongas-PR. Floresta, Curitiba, PR, n.1, jan./abr. 2005

[6] BARBIERI, J. C. Gestão Ambiental Empresarial: Conceitos, Modelos e Instrumentos. 2 ed. São Paulo: Saraiva, 2009.

[7] LEITE, P. P. S.; PIMENTA, H. C. D. Benefícios ambientais e econômicos provenientes da implementação da produção mais limpa em uma indústria de móveis de Natal-RN. Holos, Rio Grande do Norte, v.2, 2011.

[8] LEITE, B. Z.; PAWLOWSKY, U. Alternativas de minimização de resíduos em uma indústria de alimentos da região metropolitana de Curitiba. V. 10, n. 2 , abr./jun. 2005

[9] EPA - Waste Minimization Opportunity Assessmente Manual, Hazardous Waste Engineering, Office of Research and Development, U.S. Environmental Protection Agency, Cincinnati, Ohio 45268, July, 1988.

[10] MELLO, E. T. de. ; PAWLOWSKY, U. Minimização de resíduos em uma indústria de bebidas. Revista Engenharia Sanitária e Ambiental, v. 8, p. 249-256, 2003.

[11] ABNT - NBR 10.004. Resíduos sólidos e classificação. 2004

[12] MASSOTE, Carlos Henrique Ribeiro. Implementação da metodologia da produção mais limpa em uma indústria moveleira da região metropolitana de Belo Horizonte. Dissertação de mestrado. Ouro Preto, 2010.

[13] SILVA, A. F.; FIGUEIREDO, C. F. de. Reaproveitamento de resíduos de MDF da indústria moveleira. Design \& Tecnologia, UFRGS,v.1, n. 2, 2010.

[14] PEREIRA, A. F. Ecodesign: A nova ordem da indústria moveleira desafios e limites de projeto. Anais do III MADETEC - Seminário de Produtos Sólidos de Madeira de Eucalipto e Tecnologias Emergentes para a Indústria Moveleira. Universidade Federal de Viçosa, Vitória, 28 - 30 set., 2005.

[15] PEREIRA, A. F.; CARVALHO, L. de S. C. ; PINTO, A. C. de O. Resíduo de madeira: limites e possibilidades de seu uso como matéria-prima alternativa. $9^{\circ}$ Congresso Brasileiro de Pesquisa e Desenvolvimento em Design. São Paulo, 1316 out. 2010

[16] LIMA, E. G. Diagnóstico ambiental de empresas de móveis em madeira situadas no pólo moveleiro de Arapongas - PR. 2005. Dissertação (Mestrado em Engenharia Florestal) - Universidade Federal do Paraná, 2005.

[17] TORRELL, Rebeca; HILLING, Éverton; CORRADI, Giordano Marques; IWAKIRI, Setsuo. Influência da adição da serragem nas propriedades tecnológicas de painéis de madeira aglomerada de Pinus taeda. Ambiencia: Guarapuava (PR) v.9, n.1, p. 57-72, jan/abr. 2013.

[18] SILVA, S. R. da. Tijolos de solo-cimento reforçado com serragem de madeira. Dissertação, Pós-Graduação em Engenharia de Estruturas, UFMG, Belo Horizonte, 2005

[19] YAMAJI, Fábio Minoru. Produção de compósito plástico-madeira a partir de resíduos da indústria madeireira. Tese de Doutorado em Ciências Florestais. UFPR. Curitiba. 2004.

[20] ARGENTE, D. O. F. Alternativas de melhoria no processo produtivo do setor moveleiro de Santa Maria/RS: impactos ambientais. 2010. $122 \mathrm{f}$. Dissertação (Programa de Pós-Graduação mestrado em Engenharia de Produção) - Universidade Federal de Santa Maria, Santa Maria, 2010. 


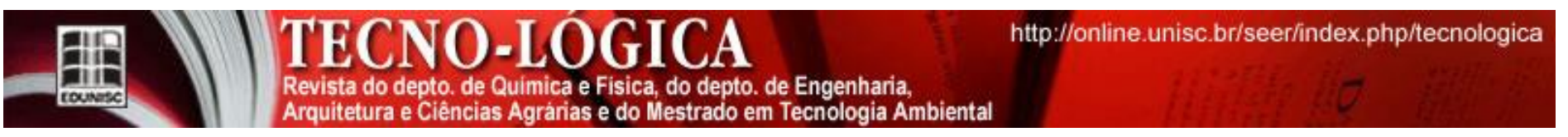

[21] SOUZA, FERREIRA, P. A. de. Recuperação de solvente dos Resíduos do processo da pintura na indústria moveleira. Dissertação de mestrado. Ouro Preto, 2009.

[22] DURSUN, D., SENGUL, F. Wastc minimization study in a solvent-based paint manufacturing plant.ResourcesConservation\&Rccycling, v.47, p. 316-331, 2006. 